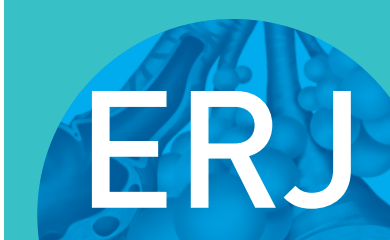

open research

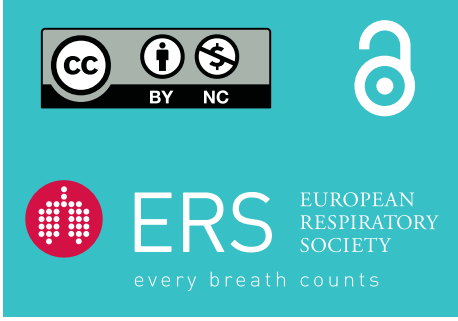

\section{Cholinergic synapse pathway gene polymorphisms associated with allergen-induced late asthmatic responses}

\section{To the Editor:}

Allergen inhalation challenge triggers well-defined airway responses in mild, allergic asthmatics. Some individuals develop only an isolated early response (early responders (ERs)) characterised by acute airway smooth muscle constriction immediately following allergen inhalation. Others develop a late response (dual responders (DRs)) that begins 3-4 h later, resulting in prolonged reduction of airway function, associated with cellular infiltration, inflammation and hyperresponsiveness of the airways [1]. It is not well understood how certain individuals are protected from developing a late response. Our previous research identified novel RNA transcripts in peripheral blood that are predictive of asthmatics who could develop a late response. Our findings pointed towards the presence of inherent differences underlying molecular mechanisms that predispose asthmatic individuals to the late response [2].

A genetic contribution to asthma aetiology has been well documented. Genetic polymorphisms have previously been shown to influence pharmacological responses in asthma [3]. To our knowledge, no studies have addressed the role of genetic variation in the allergen-induced late-phase asthmatic response. Several physiological and genetic data suggest asthmatic symptoms may be significantly modulated by the central nervous system. Changes at the level of parasympathetic neuronal control of airway smooth muscle have been shown to increase bronchoconstriction in response to vagal stimulation, leading to airway hyperresponsiveness [4]. Importantly, the cholinergic pathway mediated by the parasympathetic neurotransmitter, acetylcholine, is a predominant neurogenic mechanism contributing to bronchoconstriction in asthma [5]. We hypothesised that cholinergic pathway gene polymorphisms could play a potential role in regulating late-phase asthmatic responses after allergen inhalation.

We recruited 17 ER (six male and 11 female) and 38 DR research participants, all of whom were nonsmokers, with stable, mild allergic asthma and free of other lung diseases. The participants were of Caucasian ethnicity, and were between 18 and 55 years of age (mean \pm SD age: ER 28.47 \pm 8.88 years, DR1 $30.73 \pm 12.94$ years and DR2 $28.47 \pm 11.58$ years). Subjects who had forced expiratory volume in $1 \mathrm{~s}\left(\mathrm{FEV}_{1}\right)$ $>70 \%$ of predicted and baseline methacholine $\mathrm{PC}_{20}$ (provocative concentration causing a $20 \%$ fall in $\mathrm{FEV}_{1}$ ) of $<16 \mathrm{mg} \cdot \mathrm{mL}^{-1}$, and who developed an isolated early response $\left(\geqslant 20 \%\right.$ fall in $\mathrm{FEV}_{1}<2 \mathrm{~h}$ after allergen inhalation) or dual response (early response plus a late response: $\geqslant 15 \%$ fall in $\mathrm{FEV}_{1} 3-7 \mathrm{~h}$ after allergen inhalation) were studied. The allergens used include: ragweed, fungus, cat, grass, horse and house dust mite. The study population of DRs was split into two groups: DR1 (six male and 13 female) elicited only the dual response while DR2 (eight male and 11 female) had intermediate phenotypes (i.e. elicited different responses during repeated allergen inhalation challenge) [2]. We utilised group 1 (17 ERs and 19 DRs (DR1)) data for our original analysis and group 2 (17 ERs and 19 DRs (DR2)) to validate the results.

Blood $(5 \mathrm{~mL})$ donated by each participant was utilised for DNA extraction using standard kits (Qiagen, Hilden, Germany). A total of 140 single-nucleotide polymorphisms (SNPs) with minor allelic frequency $>10 \%$, located $<50000 \mathrm{bp}$ upstream of the transcription start site and downstream of the $3^{\prime}$ untranslated region of eight genes were selected from the cholinergic synapse KEGG (Kyoto Encyclopedia of Genes and

@ERSpublications

Cholinergic synapse pathway gene polymorphisms may play a role in regulating a type of asthmatic airway response triggered upon allergen challenge http://bit.ly/2lJx1VG

Cite this article as: Rajasekaran $\mathrm{A}, \mathrm{He} \mathrm{D}$, Yue $\mathrm{A}$, et al. Cholinergic synapse pathway gene polymorphisms associated with allergen-induced late asthmatic responses. ERJ Open Res 2019; 5: 00107-2019 [https://doi.org/10.1183/23120541.00107-2019].

Copyright $\odot$ ERS 2019. This article is open access and distributed under the terms of the Creative Commons Attribution NonCommercial Licence 4.0. 
Genomes) pathway for analysis: ADCY3, AKT3, CACNA1S, CHRM3, CHRNB2, GNB1, GNG4 and KCNQ4. Genotyping was performed using Axiom SNP arrays (Affymetrix, Santa Clara, CA, USA).

With the exception of two SNPs in CHRM3 in the DR1 group, all other SNPs satisfied Hardy-Weinberg equilibrium in both groups. A dominant genetic analysis model was adopted to assess genotype frequency distribution. Chi-squared analysis showed that the genotype and allele frequency of 28 out of 140 SNPs were significantly different between the ER and DR1 groups. This was validated in the DR2 cohort: $19(68 \%)$ out of 28 SNPs showed a significant difference between the groups. Most of these polymorphic variants within each gene were in strong linkage disequilibrium and there were no significant differences in the haplotype frequencies between the two groups in either cohort.

The cumulative effect/accumulation of minor alleles was analysed using unweighted (linear sum of minor alleles divided by total SNPs) and weighted approaches (sum of minor alleles weighted by their individual odds ratio and scaled by total SNPs). The unweighted (Uw) cumulative minor allele content (Uw-cMAC) and weighted cumulative minor allele content (w-cMAC) values of the two groups were compared using Mann-Whitney U-tests. The mean Uw-cMAC of DR1 (Uw-cMAC 25.4) was significantly higher than that of ERs (Uw-cMAC 16; $\mathrm{p}=0.001, \mathrm{z}$-score 2.99) and this was reproducible in DR2 group (Uw-cMAC 25; $\mathrm{p}=0.002$, $\mathrm{z}$-score 2.8) (figure 1a). Mean w-cMAC of DR1 (w-cMAC 24.16), but not DR2 (w-cMAC 19.47), was significantly higher than that of ERs (w-cMAC 12.18; $\mathrm{p}=0.001, \mathrm{z}$-score -3.40 ) (figure 1b).

Cholinergic pathway genes (CHRM1 and CHRM3) have been implicated as important susceptibility loci for asthma in Japanese and Mexican populations [6, 7]. Furthermore, the association of lung sensory neurons with hyperresponsive airways was shown by studies that examined the transient receptor potential $(T R P)$ gene in asthma. A recent study that examined airway hyperreactivity with a genetically silenced TRP gene in a murine model of asthma showed that ablation of vagal sensory neuronal cells abolishes hyperreactive bronchoconstrictions, even in the presence of a full lung inflammatory response [8]. A strong association between TRPA1 gene polymorphisms and childhood asthma has also been demonstrated [9].

The nicotinic acetylcholine receptor signalling mediates calcium influx via voltage-gated calcium channels (CACNA1S) that have been demonstrated to regulate airway smooth muscle contractility. In addition, the genes for G-protein coupled receptor signalling, such as ADCY3, AKT3, GNB1, GNG4 and KCNQ4, although involved in plethora of signalling pathways, have been previously associated with asthma by candidate-gene, genome-wide and epigenome-wide association studies [10-14]. Together, these genes, constituting the cholinergic synapse pathway, may mediate convergent signalling and thereby predispose an individual to a specific asthma phenotype. To our knowledge, ours is the first study showing an association of cholinergic pathway genes in allergen-induced late-phase responses. Although our small sample size limits the possibility of multiple comparisons, the rigorous clinical phenotypic characterisation and reproducibility of our findings in an additional group of DRs adds to the strength of the current study.

It is established that the early response results from IgE-mediated mast cell activation, release of mediators and subsequent bronchial smooth muscle cell constriction. In contrast, in addition to release of mediators, the late response is associated with increased airway responsiveness and inflammation [15]. The findings are consistent with our hypothesis that isolated ERs may be genetically protected from developing severe
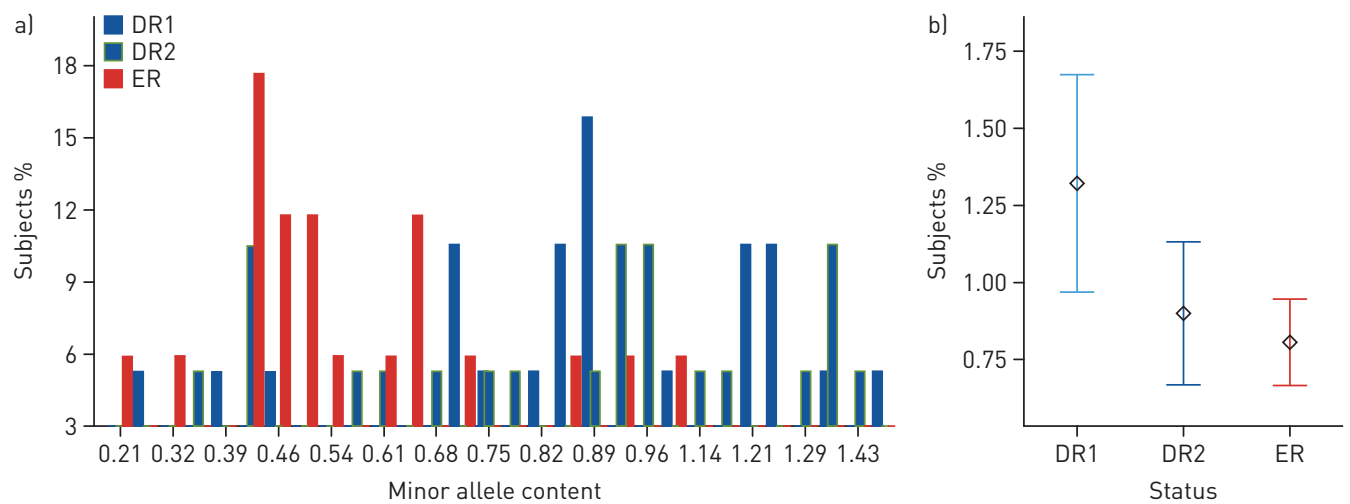

FIGURE 1 a) Minor allele distribution and b) mean differences in the weighted cumulative minor allele content (w-cMAC) between early responders (ERs) and dual responders (DRs). Error bars represent $95 \%$ confidence intervals. 
forms of airway responsiveness, while additional mechanisms could be involved in precipitating a more complex phenotype of asthma such as the late response.

For the first time, enrichment of minor allele cholinergic synapse pathway genes was demonstrated to be one of several mechanisms contributing towards late-phase responses observed in DRs. The cholinergic synapse pathway has the potential to be a therapeutic target for inflammatory phenotypes of asthma. Further elucidating its role in differentiating asthmatic responses would be a worthwhile strategy for the treatment and management of asthma.

Ashwini Rajasekaran ${ }^{1,2}$, Daniel $\mathrm{He} \oplus^{1,2}$, Alice Yue ${ }^{2,3}$, Amrit Singh $\oplus^{1,2,4}$, Casey P. Shannon $\oplus^{1,2}$, J. Mark FitzGerald ${ }^{5,6}$, Louis-Philippe Boulet ${ }^{7}$, Paul M. O'Byrne ${ }^{8}$, Gail M. Gauvreau and Scott J. Tebbutt $\oplus^{1,2,6}$

${ }^{1}$ Centre for Heart Lung Innovation, St Paul's Hospital, University of British Columbia, Vancouver, BC, Canada. ${ }^{2}$ Prevention of Organ Failure (PROOF) Centre of Excellence, Vancouver, BC, Canada. ${ }^{3}$ School of Computing Science, Simon Fraser University, Burnaby, BC, Canada. ${ }^{4}$ Dept of Pathology and Laboratory Medicine, University of British Columbia, Vancouver, BC, Canada. ${ }^{5}$ Vancouver Coastal Health Research Institute, Vancouver General Hospital, Vancouver, BC, Canada. ${ }^{6}$ Dept of Medicine, Division of Respiratory Medicine, University of British Columbia, Vancouver, BC, Canada. ${ }^{7}$ Québec Heart and Lung Institute, Laval University, Québec City, QC, Canada. ${ }^{8}$ Dept of Medicine, McMaster University, Hamilton, ON, Canada.

Correspondence: Scott J. Tebbutt, Dept of Medicine, UBC Centre for Heart Lung Innovation, Rm 166, 1081 Burrard Street, Vancouver, BC, Canada. E-mail: Scott.Tebbutt@hli.ubc.ca

Received: 29 April 2019 | Accepted after revision: 25 Aug 2019

This study is registered at www.clinicaltrials.gov with identifier number NCT01113697.

Author contributions: A. Rajasekaran, G.M. Gauvreau and S.J. Tebbutt conceived and designed the study. D. He, A. Yue, G.M. Gauvreau, J.M. FitzGerald, L-P. Boulet and P.M. O'Byrne performed the experimental procedures. A. Rajasekaran, A. Yue, A. Singh, C.P. Shannon and S.J. Tebbutt analysed and interpreted the data. A. Rajasekaran and S.J. Tebbutt prepared the manuscript.

Conflict of interest: A. Rajasekaran has nothing to disclose. D. He has nothing to disclose. A. Yue has nothing to disclose. A. Singh has nothing to disclose. C.P. Shannon has nothing to disclose. L-P. Boulet reports research grants for participation in multicentre studies from AstraZeneca, Boston Scientific, GlaxoSmithKline, Hoffman La Roche, Novartis, Ono Pharma, Sanofi and Takeda; support for investigator-initiated research projects from AstraZeneca, Boehringer Ingelheim, GlaxoSmithKline, Merck and Takeda; consulting and advisory boards for AstraZeneca, Novartis and Methapharm; author royalties from Up To Date (occupational asthma); nonprofit grants for production of educational materials from AstraZeneca, Boehringer Ingelheim, GlaxoSmithKline, Merck Frosst and Novartis; renumeration of conference fees from AstraZeneca, GlaxoSmithKline, Merck and Novartis; support for participation in conferences and meetings from Novartis and Takeda; he is a past president of the Canadian Thoracic Society and a member of its Respiratory Guidelines Committee; he is Chair of the Board of Directors of the Global Initiative for Asthma (GINA); he is Chair of the GINA Dissemination and Implementation Committee; he holds the Laval University Chair on Knowledge Transfer, Prevention and Education in Respiratory and Cardiovascular Health; he is a member of scientific committees for the American College of Chest Physicians, the American Thoracic Society, the European Respiratory Society and the World Allergy Organization; and he is the first Vice-President of the global asthma organisation InterAsma. P.M. O'Byrne reports membership of a joint oversight board for a LABA safety study; consultancy for AstraZeneca, GSK and Chiesi; and grants or grants pending from AstraZeneca, Genentech and Novartis. G.M. Gauvreau has nothing to disclose. S.J. Tebbutt has nothing to disclose.

Support statement: This research was supported by funding from AllerGen NCE Inc. (Allergy, Genes and Environment Network), PROOF Centre of Excellence, British Columbia Lung Association and BioTalent Canada.

\section{References}

1 Gauvreau GM, El-Gammal AI, O’Byrne PM. Allergen-induced airway responses. Eur Respir J 2015; 46: 819-831.

2 Singh A, Shannon CP, Kim YW, et al. Novel blood-based transcriptional biomarker panels predict the late-phase asthmatic response. Am J Respir Crit Care Med 2018; 197: 450-462.

3 Lima JJ, Zhang S, Grant A, et al. Influence of leukotriene pathway polymorphisms on response to montelukast in asthma. Am J Respir Crit Care Med 2006; 173: 379-385.

4 Belmonte KE. Cholinergic pathways in the lungs and anticholinergic therapy for chronic obstructive pulmonary disease. Proc Am Thorac Soc 2005; 2: 297-304.

5 Barnes PJ. Pharmacology of airway smooth muscle. Am J Respir Crit Care Med 1998; 158: S123-S132.

6 Maeda Y, Hizawa N, Jinushi E, et al. Polymorphisms in the muscarinic receptor 1 gene confer susceptibility to asthma in Japanese subjects. Am J Respir Crit Care Med 2006; 174.

7 Jiménez-Morales S, Jiménez-Ruíz JL, Río-Navarro BED, et al. CHRM2 but not CHRM1 or CHRM3 polymorphisms are associated with asthma susceptibility in Mexican patients. Mol Biol Rep 2014; 41: 2109-2117. 
Tränknera D, Hahnea N, Suginoa K, et al. Population of sensory neurons essential for asthmatic hyperreactivity of inflamed airways. Proc Natl Acad Sci USA 2014; 111: 11515-11520.

9 Valentina Gallo V, Dijk FN, Holloway JW, et al. TRPA1 gene polymorphisms and childhood asthma. Pediatr Allergy Immunol 2017; 28: 191-198.

10 Bradley SJ, Wiegman $\mathrm{CH}$, Maza M, et al. Mapping physiological G protein-coupled receptor signaling pathways reveals a role for receptor phosphorylation in airway contraction. Proc Natl Acad Sci USA 2016; 113: 4524-4529.

11 White $\mathrm{JH}$, Chiano $\mathrm{M}$, Wigglesworth $\mathrm{M}$, et al. Identification of a novel asthma susceptibility gene on chromosome 1qter and its functional evaluation. Hum Mol Genet 2008; 17: 1890-1903.

12 Luo W, Obeidat M, Fabio A, et al. Airway epithelial expression quantitative trait loci reveal genes underlying asthma and other airway diseases. Am J Respir Cell Mol Biol 2016; 54: 177-187.

13 Gunawardhana LP, Gibson PG, Simpson JL, et al. Characteristic DNA methylation profiles in peripheral blood monocytes are associated with inflammatory phenotypes of asthma. Epigenetics 2014; 9: 1302-1316.

14 Bai Q, Feng J, Yin Z. Abnormal DNA methylations associated with allergic asthma children. Int J Hum Genet 2018; 18: 35-42.

15 Weersink EJ, Postma DS, Aalbers R, et al. Early and late asthmatic reaction after allergen challenge. Respir Med 1994; 88: 103-114. 